\title{
Qubit detection with a T-shaped double quantum dot detector
}

\author{
JunYan Luo, , 冈 HuJun Jiao, ${ }^{2}$ Jing Hu, ${ }^{1}$ Xiao-Ling He, ${ }^{1}$ XiaoLi Lang, ${ }^{1}$ and Shi-Kuan Wang ${ }^{3}$ \\ ${ }^{1}$ Department of Physics, Zhejiang University of Science and Technology, Hangzhou 310023, China \\ ${ }^{2}$ Department of Physics, Shanxi University, Taiyuan, Shanxi 030006, China \\ ${ }^{3}$ Department of Physics, Hangzhou Dianzi University, Hangzhou 310018, China
}

(Dated: August 6, 2018)

\begin{abstract}
We propose to continuously monitor a charge qubit by utilizing a T-shaped double quantum dot detector, in which the qubit and double dot are arranged in such a unique way that the detector turns out to be particularly susceptible to the charge states of the qubit. Special attention is paid to the regime where acquisition of qubit information and backaction upon the measured system exhibit nontrivial correlation. The intrinsic dynamics of the qubit gives rise to dynamical blockade of tunneling events through the detector, resulting in a super-Poissonian noise. However, such a pronounced enhancement of detector's shot noise does not necessarily produce a rising dephasing rate. In contrast, an inhibition of dephasing is entailed by the reduction of information acquisition in the dynamically blockaded regimes. We further reveal the important impact of the charge fluctuations on the measurement characteristics. Noticeably, under the condition of symmetric junction capacitances the noise pedestal of circuit current is completely suppressed, leading to a divergent signal-to-noise ratio, and eventually to a violation of the Korotkov-Averin bound in quantum measurement. Our study offers the possibility for a double dot detector to reach the quantum limited effectiveness in a transparent manner.
\end{abstract}

PACS numbers: 03.65.Ta, 72.70.+m, 03.65.Yz, 73.23.-b

\section{INTRODUCTION}

Understanding the fundamental physics in quantum measurement process is of vital importance for physically implementing fast and efficient measurement of a two-state quantum system (qubit) [1] , as well as essential applications in quantum information processing [2, 3]. So far, a variety of mesoscopic devices have been proposed for fast readout of qubit information. For instance, a quantum point contact (QPC) has been widely investigated, with special attention paid to the nontrivial correlation between the QPC and qubit [4-14]. Alternatively, a single electron transistor (SET) was shown to have advantages over QPC in many respects, such as high sensitivity, wide circuit bandwidth, and low noise [15 21]. In particular, single-shot measurement has recently been realized based on SET detectors, in which the information of the qubit is uniquely determined in simply one run 22 25].

Historically, quantum mechanical detection was described by the projective theory, in which the measurement takes place instantaneously. In contrast, the essence of the modern theory of quantum measurement emphasizes that detector extracts information and renders the measured system in a continuous manner. The process of information acquisition from the detector and how it would alter the remaining uncertainty in the system lies at the heart of the measurement dynamics. An important figure of merit in continuous measurement is the detector "ideality" or effectiveness, characteriz-

*Electronic address: jyluo@zust.edu.cn ing how close to the quantum limit the detector could operator. In an ideal detection, qubit dephasing generated by detector backaction is purely associated with the information flow, rather than a noisy environment. For a less effective detector, qubit dephasing takes place more rapidly than the information flow. This imposes an important limit on the signal-to-noise ratio of the measurement, known as the Korotkov-Averin bound: The maximum signal-to-noise ratio the detector can reach is limited at 4 [26, 27]. It has been confirmed in Refs. [28, 29] and measured in experiment [30]. Extensions of the Korotkov-Averin bound have also been discussed in continuous measurement of coupled qubits [31, 32 and precession of an individual spin [33, 34].

For an SET detector, it usually means a single quantum dot (SQD) sandwiched between the source and drain electrodes, in which electron transport exhibits quantum coherence within the size of the reduced system. Yet, the discrete nature of the charge exhibits its inherent randomness in the process of transport. The involving shot noise and telegraph noise was recently proved to be the two sides of the same coin [35-38], and may have essential roles to play in the quantum measurement. It has been shown that the SQD detector may achieve quantum limited measurement under appropriate conditions, where qubit dephasing is due purely to the information flow, rather than detector's shot noise [21, 39]. Yet, in order to distinguish clearly the two currents corresponding to the two logical states of the qubit, it poses a very challenging condition in measurement, i.e. very low temperature.

To loosen the tough temperature restrictions, a double quantum dot (DQD) SET has recently been proposed to continuously monitor a qubit 39]. The electrostatic 

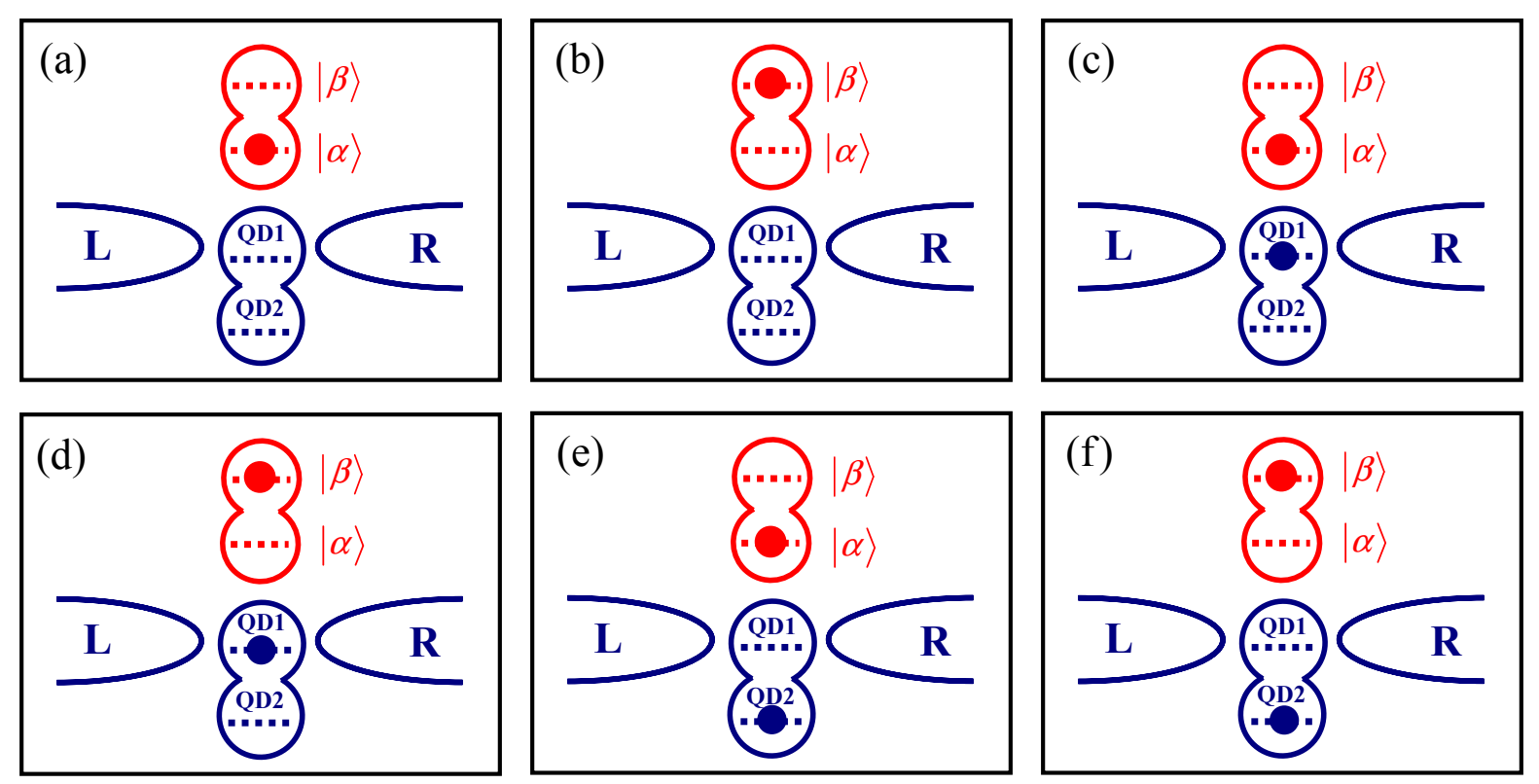

FIG. 1: Schematics of a solid-state charge qubit under the continuous measurement of a TDQD detector. Possible electron configurations of the reduced system (qubit plus TDQD) are: (a) the TDQD is empty, (c) QD1 is occupied, and (e) QD2 is occupied while the electron of the qubit resides the logic state $|\alpha\rangle$. Correspondingly, (b), (d) and (f) denote the same states but with the electron of the qubit in the state $|\beta\rangle$.

interaction between the qubit and DQD leads to an energy level mismatch between the two dots, which causes a prominent current visibility of the measurement even at a relatively high temperature. Unfortunately, its effectiveness turns out to be less than that of an ideal detector [40]. The reason is that the generated dephasing of the qubit stems partially from the detector's shot noise, such that the information of qubit encoded in the DQD detector's degree of freedom cannot be fully deduced from the measured output. It is thus appealing to find a detector capable of combining advantages of SQD and DQD detectors together, such that it could operate at a weakened temperature condition while reaching the maximum effectiveness at the same time.

In this work, we investigate this important issue in the context of a T-shaped DQD (TDQD) detector 4144], where only quantum dot 1 (QD1) is directly tunnelcoupled to the left and right electrodes, whereas quantum dot 2 (QD2) is only side-coupled to QD1 (see Fig.(1). We pay special attention to the essential correlation between the qubit and the TDQD detector. In particular, the inherent dynamics of the qubit may give rise to bunching of tunneling events though the TDQD detector, which is manifested as a pronounced super-Poissonian noise in the TDQD detector. However, such a large noise does not necessarily imply an enhancement of the dephasing rate. In contrast, the involving dynamical blockade corresponds to a no-measurement regime, where the qubit dephasing is actually suppressed. An important advantage of the SET detector is that the left and right electrodes could monitor the qubit simultaneously, such that any noise not shared by two electrodes can be filtered out, making it analogous to the measurement setup of twin quantum point contacts [45]. However, the crucial difference is that the currents through the left and right junctions of the TDQD detector are intrinsically correlated to each other via the charge fluctuations in the TDQD. We demonstrate that although the signal-to-noise ratio associated with the junction noise alone could not approach the quantum limit, the spectrum of charge fluctuations in the TDQD results in a complete suppression of the the noise pedestal, leading eventually to a divergent signalto-noise ratio and thus a violation of the Korotkov-Averin bound.

The paper is organized as follows. We start in Sec.[II with a description the measurement setup and corresponding Hamiltonian for this scenario. The particlenumber-resolved quantum master equation (QME) to the reduced dynamics and measurement characteristics is outlined in Sec.III. The influence of qubit dynamics on the TDQD detector shot noise is analyzed in Sec.IV. which is then followed by the discussion qubit dephasing behavior associated with detector's output in Sec. D Sec.VI is focused on the measurement effectiveness of the TDQD detector in terms of the signal-to-noise ratio, with special attention paid to the violation of the KorotkovAverin bound. Finally, we conclude in Sec.VII 
TABLE I: The eigenenergies and corresponding eigenstates of the reduced system (qubit plus TDQD) for $\epsilon_{\mathrm{q}}=\epsilon_{\mathrm{T}}=0$, and $\Omega$, $W \ll U$.

\begin{tabular}{ccc}
\hline \hline$N_{\text {tot }}$ & Eigenenergy & Eigenstate \\
\hline 1 & $E_{1}=-\Omega$ & $|1\rangle=\frac{1}{\sqrt{2}}(|\mathrm{a}\rangle-|\mathrm{b}\rangle)$ \\
2 & $E_{2}=+\Omega$ & $|2\rangle=\frac{1}{\sqrt{2}}(|\mathrm{a}\rangle+|\mathrm{b}\rangle)$ \\
3 & $E_{3} \simeq 0$ & $|3\rangle=\frac{1}{\sqrt{2}}(|\mathrm{~d}\rangle-|\mathrm{e}\rangle)$ \\
4 & $E_{4} \simeq U$ & $|4\rangle \simeq|\mathrm{c}\rangle$ \\
5 & $E_{5} \simeq+\frac{1}{\sqrt{2}}(\Omega+W)$ & $|5\rangle \simeq \frac{1}{2}(|\mathrm{~d}\rangle+|\mathrm{e}\rangle+\sqrt{2}|\mathrm{f}\rangle)$ \\
6 & $E_{6} \simeq-\frac{1}{\sqrt{2}}(\Omega+W)$ & $|6\rangle \simeq \frac{1}{2}(|\mathrm{~d}\rangle+|\mathrm{e}\rangle-\sqrt{2}|\mathrm{f}\rangle)$ \\
\hline \hline
\end{tabular}

\section{MODEL DESCRIPTION}

The system under study is shown schematically in Fig.11. The charge qubit is represented by an extra electron in a double quantum dot. Whenever the electron occupies the lower (upper) dot, the qubit is said to be in the logic state $|\alpha\rangle(|\beta\rangle)$. The detector is a TDQD SET, in which QD1 is directly tunnel-coupled to the left (L) and right $(\mathrm{R})$ electrodes, whereas QD2 is only side-coupled to QD1. We assume that each quantum dot has only one level involved in transport within the bias window defined by the Fermi levels of the left and right electrodes. Furthermore, both interdot and intradot charging energies are much larger than the Fermi levels such that at most one electron can reside on the TDQD. The Hilbert space of the TDQD dot is thus reduced to $|0\rangle$-empty, $|1\rangle$ $(|2\rangle)$-one electron in QD1 (QD2). The qubit is placed in vicinity of QD1, as shown in Fig.1. Under such a unique arrangement the measured current is expected to be particularly susceptible to electron configurations of the qubit. It is right this mechanism that can be utilized to sensitively acquire the qubit-state information from the output of the TDQD detector.

The entire system Hamiltonian reads

$$
H=H_{\mathrm{S}}+H_{\mathrm{B}}+H^{\prime} .
$$

The first part denotes the Hamiltonian of the reduced system (qubit plus TDQD)

$$
H_{\mathrm{S}}=\frac{1}{2} \epsilon_{\mathrm{q}} \sigma_{z}+\Omega \sigma_{x}+\frac{1}{2} \epsilon_{\mathrm{T}} Q_{z}+W Q_{x}+U|\alpha\rangle\langle\alpha|\otimes| 1\rangle\langle 1|,
$$

where we have introduced pseudo-spin operators $\sigma_{z} \equiv$ $|\alpha\rangle\langle\alpha|-| \beta\rangle\left\langle\beta\left|, \sigma_{x} \equiv\right| \alpha\right\rangle\langle\beta|+| \beta\rangle\langle\alpha|$ for the qubit, and likewise for the TDQD $Q_{z} \equiv|1\rangle\langle 1|-| 2\rangle\langle 2|, Q_{x} \equiv$ $|1\rangle\langle 2|+| 2\rangle\langle 1|$. The level detuning and interdot coupling in the qubit (TDQD) are $\epsilon_{\mathrm{q}}\left(\epsilon_{\mathrm{T}}\right)$ and $\Omega_{\mathrm{q}}(W)$, respectively. The qubit is placed in close proximity to the QD1, such that the energy level of QD1 is very sensitive to the qubit occupations, as represented by the last term in Eq. (2). There are totally six possible electron configurations of the reduced system (qubit plus TDQD), as shown in Fig.11(a)-(f). Let $\{|\mathrm{a}\rangle, \cdots,|\mathrm{f}\rangle\}$ be the states of the reduced system corresponding to charge configura- tions in Fig.1(a)-(f). The eigenenergies and corresponding eigenstates of the reduced system are listed in Table I for $\epsilon_{\mathrm{q}}=\epsilon_{\mathrm{T}}=0$, and $\Omega, W \ll U$.

The electrodes are modeled as reservoirs of noninteracting electrons

$$
H_{\mathrm{B}}=\sum_{\ell=\mathrm{L}, \mathrm{R}} \sum_{k} \varepsilon_{\ell k} c_{\ell k}^{\dagger} c_{\ell k},
$$

where $c_{\ell k}^{\dagger}\left(c_{\ell k}\right)$ stands for the creation (annihilation) operator for an electron with momentum $k$ in the left $(\ell=\mathrm{L})$ or right $(\ell=\mathrm{R})$ electrode. The left/right electron reservoir is characterized by the Fermi distribution $f_{\mathrm{L} / \mathrm{R}}(\omega)$. The voltage is symmetrically applied, which leads to symmetric Fermi levels in the left and right electrodes, i.e. $\mu_{\mathrm{L} / \mathrm{R}}= \pm e V / 2$.

Electron tunneling between the QD1 and electrodes is described by

$$
H^{\prime}=\sum_{\ell, k}\left(t_{\ell k} c_{\ell k}^{\dagger}|0\rangle\langle 1|+\text { h.c. }\right) \equiv \sum_{\ell}\left(f_{\ell}|0\rangle\langle 1|+\text { h.c. }\right),
$$

where $f_{\ell} \equiv \sum_{k} t_{\ell k} c_{\ell k}^{\dagger}$. The tunnel-coupling strength between electrode $\ell=\{\mathrm{L}, \mathrm{R}\}$ and QD1 is given by the intrinsic tunneling width $\Gamma_{\ell}(\omega)=2 \pi \sum_{k}\left|t_{\ell k}\right|^{2} \delta\left(\omega-\varepsilon_{\ell k}\right)$. Hereafter, we consider wide band in the electrodes, which results in energy independent couplings $\Gamma_{\mathrm{L} / \mathrm{R}}$. The total tunneling width is thus given by $\Gamma=\Gamma_{L}+\Gamma_{R}$. The effects of stochastic electron reservoirs on the measurement are characterized by the bath correlation functions

$$
\begin{aligned}
& C_{\ell}^{(+)}(t-\tau)=\left\langle f_{\ell}^{\dagger}(t) f_{\ell}(\tau)\right\rangle_{\mathrm{B}}, \\
& C_{\ell}^{(-)}(t-\tau)=\left\langle f_{\ell}(t) f_{\ell}^{\dagger}(\tau)\right\rangle_{\mathrm{B}},
\end{aligned}
$$

where $\langle\cdots\rangle_{\mathrm{B}} \equiv \operatorname{tr}_{\mathrm{B}}\left[(\cdots) \rho_{\mathrm{B}}\right]$ stands for the trace over degrees of freedom of the electron reservoirs, with $\rho_{\mathrm{B}}$ the local thermal equilibrium state of the electrodes. Throughout this work, we set $\hbar=e=1$ for the Planck constant and electron charge, unless stated otherwise.

\section{PARTICLE-NUMBER-RESOLVED QUANTUM MASTER EQUATION APPROACH}

The stochastic process of electron tunneling through the TDQD detector may be characterized by the joint 


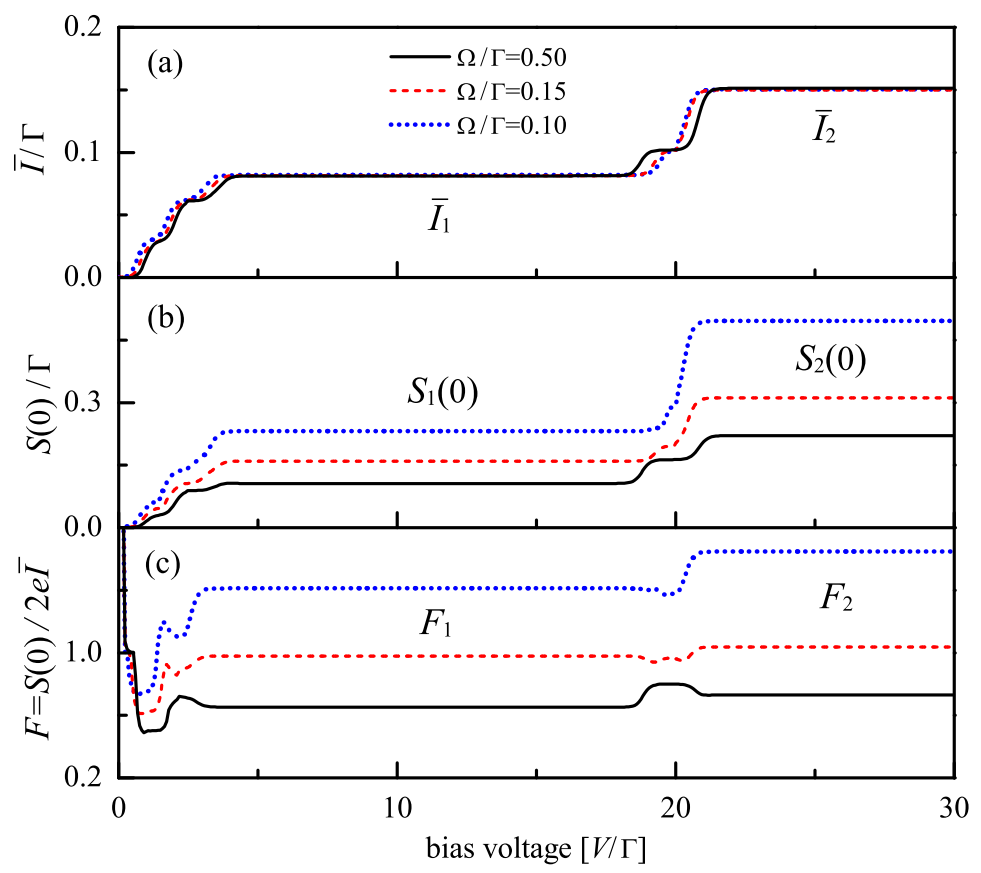

FIG. 2: (a) The measurement current $\bar{I}$, (b) zero frequency noise $S(0)$, and (c) Fano factor $F \equiv$ $S(0) /(2 e \bar{I})$ versus the bias voltage for different values of $\Omega$. Each time when the Fermi level of the electrode aligns with one of the excitation energies as indicated in Table 【, a new transport channel opens. This leads to plateaus, separated by thermally broadened steps. The current and noise are measured in unit of $\Gamma \equiv \Gamma_{\mathrm{L}}+\Gamma_{\mathrm{R}}$. Other plotting parameters are: $\epsilon_{\mathrm{q}}=\epsilon_{\mathrm{T}}=0, W / \Gamma=$ $0.5, k_{\mathrm{B}} T / \Gamma=2.0, \Gamma_{\mathrm{L}} / \Gamma_{\mathrm{R}}=1 / 3$, and $U / \Gamma=10$.

probability distribution $P\left(N_{\mathrm{L}}, N_{\mathrm{R}}, t\right)$ of finding $N_{\mathrm{L}}$ electrons transmitted thought the left junction and $N_{\mathrm{R}}$ electrons tunneled thought the right one in the given time $t$. Alternatively, it can be described by the current cumulants, known as full counting statistics [46, 47], which provides a unique signature of measurement characteristics. For that purpose, we employ a particle-number-resolved reduced density matrix $\rho^{\left(N_{\mathrm{L}}, N_{\mathrm{R}}\right)}$ for specific number of $N_{\mathrm{L}}\left(N_{\mathrm{R}}\right)$ electrons passed through the left (right) junc- tion. The corresponding particle-number-resolved QME reads [48 4 4]

$$
\dot{\rho}^{\left(N_{\mathrm{L}}, N_{\mathrm{R}}\right)}=-\mathrm{i} \mathcal{L} \rho^{\left(N_{\mathrm{L}}, N_{\mathrm{R}}\right)}-\left\{\mathcal{R}_{0}+\mathcal{R}_{\mathrm{L}}+\mathcal{R}_{\mathrm{R}}\right\} \rho^{\left(N_{\mathrm{L}}, N_{\mathrm{R}}\right)},
$$

where $\mathcal{L}(\cdots) \equiv\left[H_{\mathrm{S}},(\cdots)\right]$ is the Liouvillian associated with the reduced system (qubit plus TDQD) Hamiltonian,

$$
\mathcal{R}_{0} \rho^{\left(N_{\mathrm{L}}, N_{\mathrm{R}}\right)}=\frac{1}{2}\left\{|1\rangle\left\langle 0\left|A^{(-)} \rho^{\left(N_{\mathrm{L}}, N_{\mathrm{R}}\right)}+\rho^{\left(N_{\mathrm{L}}, N_{\mathrm{R}}\right)} A^{(+)}\right| 1\right\rangle\langle 0|\right\}+\text { h.c. }
$$

describes the continuous evolution of the reduced system, whereas

$$
\mathcal{R}_{\mathrm{L}} \rho^{\left(N_{\mathrm{L}}, N_{\mathrm{R}}\right)}=-\frac{1}{2}\left\{A_{\mathrm{L}}^{(-)} \rho^{\left(N_{\mathrm{L}}-1, N_{\mathrm{R}}\right)}|1\rangle\langle 0|+| 1\rangle\langle 0| \rho^{\left(N_{\mathrm{L}}+1, N_{\mathrm{R}}\right)} A_{\mathrm{L}}^{(+)}\right\}+\text {h.c. }
$$

and

$$
\mathcal{R}_{\mathrm{R}} \rho^{\left(N_{\mathrm{L}}, N_{\mathrm{R}}\right)}=-\frac{1}{2}\left\{A_{\mathrm{R}}^{(-)} \rho^{\left(N_{\mathrm{L}}, N_{\mathrm{R}}-1\right)}|1\rangle\langle 0|+| 1\rangle\langle 0| \rho^{\left(N_{\mathrm{L}}, N_{\mathrm{R}}+1\right)} A_{\mathrm{R}}^{(+)}\right\}+\text {h.c. }
$$

represent jumps of electrons via the left and right electrodes, respectively. Here $A^{( \pm)}=\sum_{\ell} A_{\ell}^{( \pm)}$, with $A_{\ell}^{( \pm)} \equiv$ $C_{\ell}^{( \pm)}( \pm \mathcal{L})(|0\rangle\langle 1|)$. The involving reservoir spectral functions are defined as the Fourier transform of the reservoir correlation functions

$$
C_{\ell}^{( \pm)}( \pm \mathcal{L})=\int_{-\infty}^{\infty} \mathrm{d} t C_{\ell}^{( \pm)}(t) e^{ \pm \mathrm{i} \mathcal{L} t}
$$

The particle-number-resolved quantum master equation (6) provides us direct access to the joint probability distribution for the number of particles transmitted through the left and right junctions, i.e. $P\left(N_{\mathrm{L}}, N_{\mathrm{R}}, t\right)=$ $\operatorname{tr}\left\{\rho^{\left(N_{\mathrm{L}}, N_{\mathrm{R}}\right)}(t)\right\}$, where $\operatorname{tr}\{\cdots\}$ represents the trace over the degrees of freedom of the reduced system (qubit plus TDQD). The first cumulant of the probability distribution corresponds to the current through the left 
TABLE II: The stationary current and Fano factor for $\epsilon_{\mathrm{q}}=\epsilon_{\mathrm{T}}=0$, and $\Omega, W \ll U$ in the bias regime 1 : $2\left(E_{4}-E_{2}\right)>$ $V>2\left(E_{5}+E_{2}\right)$ and regime $2: V>2\left(E_{4}+E_{2}\right)$. Owing to symmetric application of the bias voltage, the steps and hence the different bias regimes are distinguished at twice of the excitation energies as indicated in Table $\square$

\begin{tabular}{|c|c|c|}
\hline$i$ & bias regime 1 & bias regime 2 \\
\hline $\bar{I}_{i}$ & $\frac{\Gamma_{\mathrm{L}} \Gamma_{\mathrm{R}}}{3 \Gamma_{\mathrm{L}}+2 \Gamma_{\mathrm{R}}}$ & $\frac{\Gamma_{L} \Gamma_{R}}{2 \Gamma_{L}+\Gamma_{R}}$ \\
\hline$F_{i}$ & $\frac{\Gamma_{\mathrm{L}}^{2}+4 \Gamma_{\mathrm{R}}^{2}}{\left(3 \Gamma_{\mathrm{L}}+2 \Gamma_{\mathrm{R}}\right)^{2}}+\frac{\Gamma_{\mathrm{L}}^{2} \Gamma_{\mathrm{R}}^{2}\left(W^{2}+4 \Omega^{2}\right)+4 \Gamma_{\mathrm{L}}^{2}\left(W^{4}+4 \Omega^{4}\right)}{2\left(3 \Gamma_{\mathrm{L}}+2 \Gamma_{\mathrm{R}}\right)^{2} \Omega^{2} W^{2}}$ & $\frac{4 \Gamma_{\mathrm{L}}^{2}+\Gamma_{\mathrm{R}}^{2}}{\left(2 \Gamma_{\mathrm{L}}+\Gamma_{\mathrm{R}}\right)^{2}}+\frac{2 \Gamma_{\mathrm{L}}^{2}\left(\Gamma_{\mathrm{R}}^{2}+8 \Omega^{2}\right) \Omega^{2}+\Gamma_{\mathrm{L}}^{2}\left(\Gamma_{\mathrm{L}}^{2}+2 W^{2}\right) W^{2}}{\left(2 \Gamma_{\mathrm{L}}+\Gamma_{\mathrm{R}}\right)^{2} \Omega^{2} W^{2}}$ \\
\hline
\end{tabular}

$(\ell=\mathrm{L})$ or right $(\ell=\mathrm{R})$ junction, given by $I_{\ell}=$ $\frac{\mathrm{d}}{\mathrm{d} t} \sum_{N_{\mathrm{L}}, N_{\mathrm{R}}} N_{\ell} P\left(N_{\mathrm{L}}, N_{\mathrm{R}}\right)=\operatorname{tr}\left\{\frac{\mathrm{d}}{\mathrm{d} t} \hat{N}_{\ell}\right\}$, where $\hat{N}_{\ell} \equiv$ $\sum_{N_{\mathrm{L}}, N_{\mathrm{R}}} N_{\ell} \rho^{\left(N_{\mathrm{L}}, N_{\mathrm{R}}\right)}$ can be evaluated via its equation of motion

$$
\frac{\mathrm{d}}{\mathrm{d} t} \hat{N}_{\ell}=-\mathrm{i} \mathcal{L} \hat{N}_{\ell}-\mathcal{R} \hat{N}_{\ell}+\mathcal{T}_{\ell}^{(-)} \rho
$$

with

$$
\begin{gathered}
\mathcal{R}(\cdots)=\frac{1}{2}\left[|1\rangle\langle 0|, A^{(-)}(\cdots)-(\cdots) A^{(+)}\right]+\text {h.c. } \quad(9 \mathrm{~b}) \\
\mathcal{T}_{\ell}^{( \pm)}(\cdots)=\frac{1}{2}\left[A_{\ell}^{(-)}(\cdots)|1\rangle\langle 0| \pm| 1\rangle\langle 0|(\cdots) A_{\ell}^{(+)}\right]+\text {h.c.. }
\end{gathered}
$$

Straightforwardly, the measured current through junction $\ell$ is given by

$$
I_{\ell}(t)=\operatorname{tr}\left\{\mathcal{T}_{\ell}^{(-)} \rho(t)\right\}
$$

where $\rho(t)$ is the unconditional density matrix that satisfies

$$
\dot{\rho}=-\mathrm{i} \mathcal{L} \rho-\mathcal{R} \rho
$$

The second cumulant of the probability distribution is directly related to the shot noise. Here, we focus on the noise spectrum of circuit current. According to the Ramo-Shockley theorem [46], the circuit current is given by $I(t)=\eta_{\mathrm{L}} I_{\mathrm{L}}+\eta_{\mathrm{R}} I_{\mathrm{R}}$. Here $\eta_{\mathrm{L}}$ and $\eta_{\mathrm{R}}$ are coefficients related to the junction capacitances that satisfy $\eta_{\mathrm{L}}+\eta_{\mathrm{R}}=$ 1 [46]. The transport currents through the left and right junctions are actually fluctuating in time, which give rise to charge accumulation " $Q$ " on the TDQD. Due to charge conservation, it simply yields

$$
\dot{Q}=I_{\mathrm{L}}-I_{\mathrm{R}}
$$

One readily obtains the correlation function of circuit current

$I(t) I(0)=\eta_{\mathrm{L}} I_{\mathrm{L}}(t) I_{\mathrm{L}}(0)+\eta_{\mathrm{R}} I_{\mathrm{R}}(t) I_{\mathrm{R}}(0)-\eta_{\mathrm{L}} \eta_{\mathrm{R}} \dot{Q}(t) \dot{Q}(0)$

As a result, the noise spectrum of circuit current consists of the following three parts [55, 56]

$$
S(\omega)=\eta_{\mathrm{L}} S_{\mathrm{L}}(\omega)+\eta_{\mathrm{R}} S_{\mathrm{R}}(\omega)-\eta_{\mathrm{L}} \eta_{\mathrm{R}} S_{\mathrm{ch}}(\omega),
$$

where $S_{\mathrm{L}}\left(S_{\mathrm{R}}\right)$ is the noise spectrum of the left (right) junction current, whereas $S_{\mathrm{ch}}(\omega)$ stands for charge fluctuations in the TDQD.

The noise spectrum of tunneling current $S_{\ell}(\omega) \quad(\ell=\mathrm{L}$ or R) may be evaluated via the MacDonald's formula 57, 58.

$$
S_{\ell}(\omega)=\omega \int_{0}^{\infty} \mathrm{d} t \sin (\omega t) \frac{\mathrm{d}}{\mathrm{d} t}\left[\left\langle\hat{N}_{\ell}^{2}(t)\right\rangle-(\bar{I} t)^{2}\right] .
$$

Hereafter, it is assumed that the reduced system evolves from $t_{0}=-\infty$, such that reduced state at $t=0$, when measurement begins, have reached the stationary state $\rho_{\text {st }}$. The involving current thus is a stationary one, i.e. $\bar{I}=I(t \rightarrow \infty)$. By employing the particlenumber-resolved quantum master equation (6), the quantity $\left\langle\hat{N}_{\ell}^{2}(t)\right\rangle \equiv \operatorname{tr}\left\{\sum_{N_{\mathrm{L}}, N_{\mathrm{R}}} N_{\ell}^{2} \rho^{\left(N_{\mathrm{L}}, N_{\mathrm{R}}\right)}\right\}$ is simply given by

$$
\frac{\mathrm{d}}{\mathrm{d} t}\left\langle\hat{N}_{\ell}^{2}(t)\right\rangle=\operatorname{tr}\left\{2 \mathcal{T}_{\ell}^{(-)} \hat{N}_{\ell}(t)+\mathcal{T}_{\ell}^{(+)} \rho_{\mathrm{st}}\right\}
$$

where $\hat{N}_{\ell}(t)$ is obtained from Eq. (9).

For the charge fluctuations in the TDQD, the symmetrized spectrum reads 55 ]

$$
S_{\mathrm{ch}}(\omega)=\omega^{2} \int_{-\infty}^{\infty} \mathrm{d} \tau\langle Q(\tau) Q+Q Q(\tau)\rangle e^{\mathrm{i} \omega \tau},
$$

where $Q \equiv|1\rangle\langle 1|+| 2\rangle\langle 2|$ stands for the operator of electron charge on the TDQD, and $\langle Q(\tau) Q\rangle \equiv$ $\operatorname{tr}\left\{\operatorname{tr}_{\mathrm{B}}\left[U^{\dagger}(\tau) Q U(\tau) Q \rho_{\mathrm{st}} \rho_{\mathrm{B}}\right]\right\}$, with $U(\tau)$ being the evolution operator associated with the entire system Hamiltonian (11). By introducing an alternative reduced density matrix $\tilde{\rho}(\tau) \equiv \operatorname{tr}_{\mathrm{B}}\left[U(\tau) Q \rho_{\mathrm{st}} \rho_{\mathrm{B}} U^{\dagger}(\tau)\right]$, the charge correlation can be further reduced to $\langle Q(\tau) Q\rangle=\operatorname{tr}\{Q \tilde{\rho}(\tau)\}$. Under the second-order Born-Markov approximation, it is found that $\tilde{\rho}(t)$ satisfies the same equation as $\rho(t)$ in Eq. (11), with the only crucial difference of the initial condition $\tilde{\rho}(0)=Q \rho_{\text {st }}$. Eventually, the noise spectrum of charge fluctuations reads

$$
S_{\mathrm{ch}}(\omega)=2 \omega^{2} \operatorname{Re}\{\operatorname{tr}[Q \tilde{\rho}(\omega)+Q \tilde{\rho}(-\omega)]\},
$$

where $\tilde{\rho}(\omega)$ is the Fourier transform of $\tilde{\rho}(t)$ and satisfies

$$
-\mathrm{i} \omega \tilde{\rho}(\omega)=-\mathrm{i} \mathcal{L} \tilde{\rho}(\omega)-\mathcal{R} \tilde{\rho}(\omega)+Q \rho_{\text {st }} .
$$



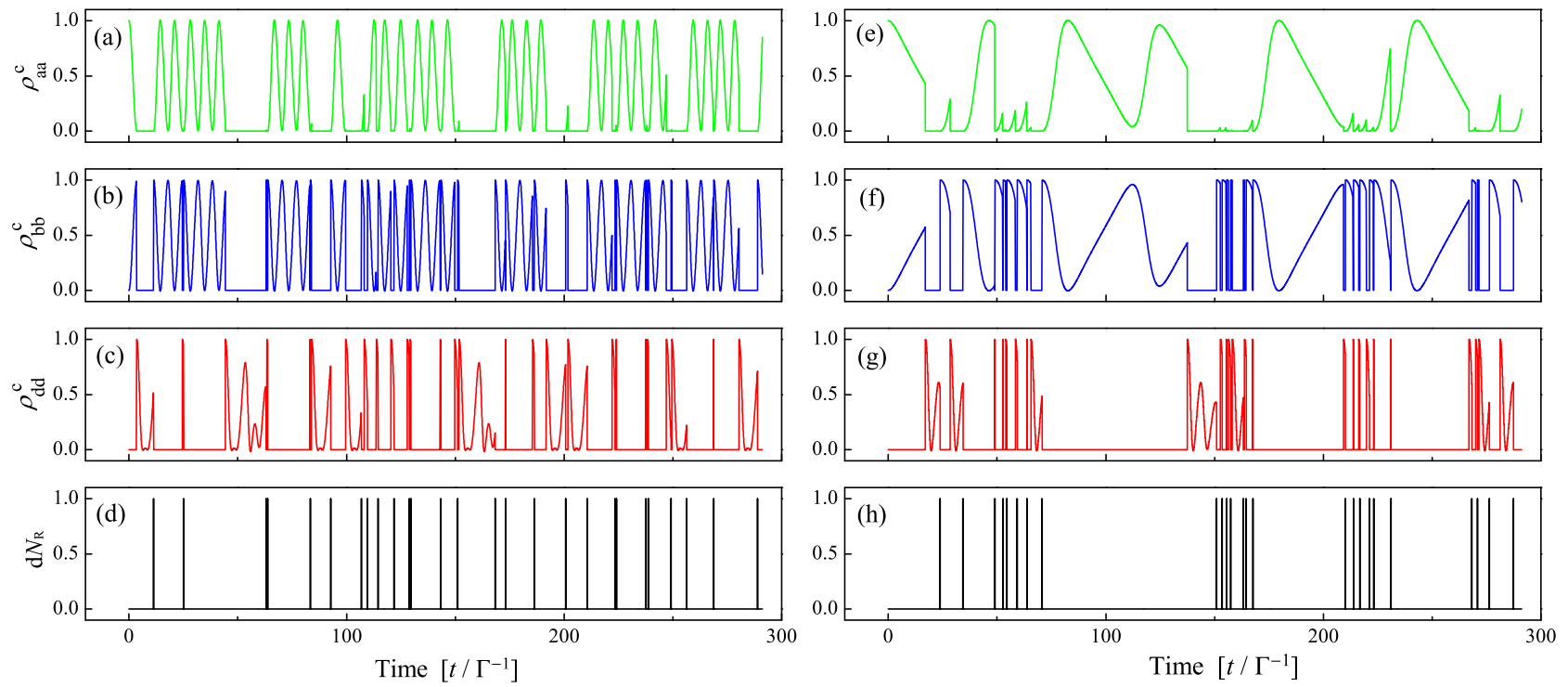

FIG. 3: Sets of typical quantum trajectories and corresponding detection records for $\Omega / \Gamma=0.5(\mathrm{a})-(\mathrm{d})$ and $\Omega / \Gamma=0.1(\mathrm{e})-(\mathrm{h})$ with the same initial condition $\rho_{\mathrm{aa}}^{\mathrm{c}}(t=0) \equiv\left\langle\mathrm{a}\left|\rho^{\mathrm{c}}(t=0)\right| \mathrm{a}\right\rangle=1$. The measurement voltage $V / \Gamma=10$ is within the bias regime 1 [see Fig.2], such that the state "|c)" as shown in Fig.11(c) is energetically prohibited. The Fermi energies are far from the excitation energies of the reduced system, such that the Fermi functions can be approximated by either 1 or 0 , and thus temperature is not involved here. The time step used is $\Delta t=0.01 \Gamma^{-1}$. Other plotting parameters are: $\epsilon_{\mathrm{q}}=\epsilon_{\mathrm{T}}=0, W / \Gamma=0.5$, $\Gamma_{\mathrm{L}} / \Gamma_{\mathrm{R}}=1 / 3$, and $U / \Gamma=10$.

\section{QUBIT DYNAMICS INDUCED SUPER-POISSONIAN NOISE}

The measurement current $\bar{I}$, zero frequency noise $S(0)$, and the Fano factor $F=S(0) /(2 e \bar{I})$ versus voltage are plotted in Fig.2(a)-(c), respectively. At very low bias $V \ll k_{\mathrm{B}} T$, electron transport through the TDQD detector is exponentially suppressed. The current fluctuation is dominated by thermal noise described by the hyperbolic cotangent behavior [46], which leads to a divergence of the Fano factor at $V=0$, as shown in Fig.2(c). Each time when a new excitation energy (as indicated in Table 【i) lies within the energy window defined by the chemical potentials of the left and right electrodes, a new transport channel opens, which gives rise to plateaus, separated by thermally broadened steps. Owing to symmetric application of the bias, the steps take place at bias voltages twice of the corresponding excitation energy.

The plateau heights of the current are found to be independent on $\Omega$. Variation of $\Omega$ changes the eigenenergies (see Table 【), leading thus only to small shift of the current steps, as displayed in Fig.2(a). The plateau heights of noise and Fano factor, however, are sensitively modulated by $\Omega$, showing shot noise as a more sensitive diagnostic tool than the current. For $\Omega / \Gamma=0.5$, the noise is well below the Poissonian value. An decrease in $\Omega$ leads to a strong enhancement of the Fano factor. In particular, a prominent super-Poissonian noise is observed for $\Omega / \Gamma=0.1$, as shown by the dotted curve in Fig. 2 (c). In literature, different mechanisms responsible for super-
Poissonian noise have been identified, such as dynamical channel blockade 59 62], dynamical spin blockade 63 65], or cotunneling events 66 68]. Our result reveals that the intrinsic dynamics of the qubit serves as an additional mechanism that may lead to super-Poissonian shot noise in a double dot detector.

Specifically, let us investigate the current and noise in the bias regime 1: $2\left(E_{4}-E_{2}\right)>V>2\left(E_{5}+E_{2}\right)$ and regime $2: V>2\left(E_{4}+E_{2}\right)$. Here the factor of "2" arises from the symmetric application of the bias voltage. In these two wide regions where electrode chemical potentials are far away from the excitation energies of the double dot, the Fermi functions can be well approximated by either one or zero. Analytical results of the current and noise are obtained for $\epsilon_{\mathrm{q}}=\epsilon_{\mathrm{T}}=0$, and $\Omega, W \ll U$, as listed in Table III Indeed, the current plateau height depend on the coupling parameters $\Gamma_{L}$ and $\Gamma_{R}$ only, while noise and Fano factor are both sensitive to the interdot couplings $\Omega$ and $W$. Strikingly, a divergent Fano factor is found in the limit $\Omega \rightarrow 0$ or $W \rightarrow 0$.

To investigate in detail the underlying physics that leads to the divergent Fano factor, we now resort to the real-time measurement dynamics of the reduced system, i.e., the usual single measurement realizations in experiments. In what follows, we will consider a typical voltage $V / \Gamma=10$ in the bias regime 1 as shown in Fig.2, such that the state "|c|" [Fig.1(c)] is energetically prohibited. The reason we consider this regime is that the measured current visibility, defined as $\left|I_{\alpha}-I_{\beta}\right| /\left(I_{\alpha}+I_{\beta}\right)$, can reach the maximum value of 1 . Here $I_{\alpha}\left(I_{\beta}\right)$ stands for the 

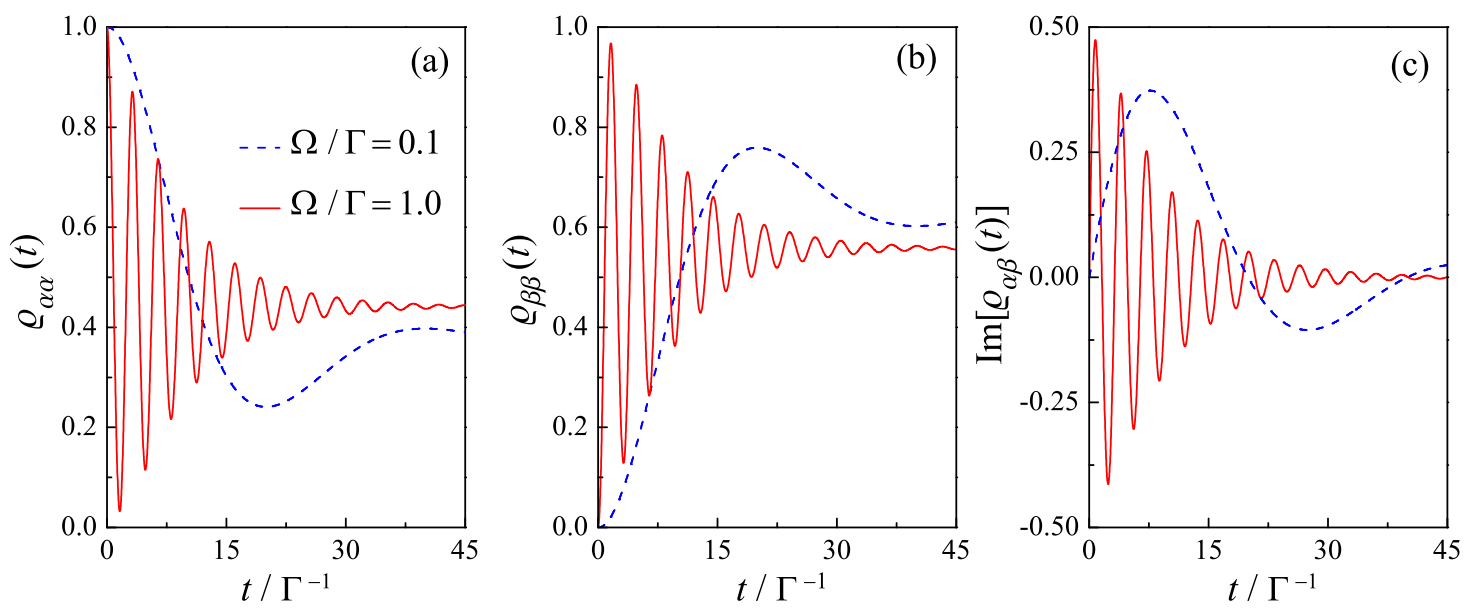

FIG. 4: Measurement-induced qubit relaxation and dephasing versus time for $\Omega / \Gamma=0.1$ (dashed curves) and $\Omega / \Gamma=1.0$ (solid curves). (a) $\varrho_{\alpha \alpha}(t)$, (b) $\varrho_{\beta \beta}(t)$, (c) the imaginary part of the off-diagonal element $\varrho_{\alpha \beta}$. The total tunneling width $\Gamma=\Gamma_{\mathrm{L}}+\Gamma_{\mathrm{R}}$ is kept constant, and interdot hopping in the TDQD is $W / \Gamma=1.0$. The qubit is assumed to be symmetric $\left(\epsilon_{\mathrm{q}}=0\right)$ and initially in the logical state $|\alpha\rangle$, i.e. $\varrho(t=0)=|\alpha\rangle\langle\alpha|$. Other plotting parameters used are: $V / \Gamma=10, \epsilon_{\mathrm{T}}=0, \Gamma_{\mathrm{L}} / \Gamma_{\mathrm{R}}=1 / 4$ and $U / \Gamma=10$.

current through the TDQD when the qubit occupies the logical state $|\alpha\rangle(|\beta\rangle)$. Electrons flow in one direction: An extra electron injects into the QD1 from the left electrode, dwells in the double dot for a certain amount of time before it escapes to the right electrode. We introduce two stochastic point variables $\mathrm{d} N_{\mathrm{L}}(t)$ and $\mathrm{d} N_{\mathrm{R}}(t)$ (with values either 0 or 1 ) to represent, respectively, the number of electron tunneled into QD1 from the left electrode and that escaped to the right electrode from the $\mathrm{QD1}$, during the infinitesimal time interval $\mathrm{d} t$. According to the quantum trajectory theory, the evolution of the reduced system is given by the following conditional QME [69]

$$
\begin{aligned}
\mathrm{d} \rho^{\mathrm{c}}= & -\mathrm{i} \mathcal{L} \rho^{\mathrm{c}}(t) \mathrm{d} t-\left\{\Gamma_{\mathrm{L}} \mathcal{A}[|1\rangle\langle 0|]+\Gamma_{\mathrm{R}} \mathcal{A}[|0\rangle\langle 1|]-\mathcal{P}_{\mathrm{L}}(t)-\mathcal{P}_{\mathrm{R}}(t)\right\} \rho^{\mathrm{c}}(t) \mathrm{d} t \\
& +\mathrm{d} N_{\mathrm{L}}\left[\frac{\mathcal{J}\left[\sqrt{\Gamma_{\mathrm{L}}}|1\rangle\langle 0|\right]}{\mathcal{P}_{\mathrm{L}}(t)}-1\right] \rho^{\mathrm{c}}(t)+\mathrm{d} N_{\mathrm{R}}\left[\frac{\mathcal{J}\left[\sqrt{\Gamma_{\mathrm{R}}}|0\rangle\langle 1|\right]}{\mathcal{P}_{\mathrm{R}}(t)}-1\right] \rho^{\mathrm{c}}(t),
\end{aligned}
$$

where we have introduced the superoperators $\mathcal{J}[X] \rho^{\mathrm{c}} \equiv$ $X \rho^{\mathrm{c}} X^{\dagger}$ and $\mathcal{A}[X] \rho^{\mathrm{c}} \equiv \frac{1}{2}\left(X^{\dagger} X \rho^{\mathrm{c}}+\rho^{\mathrm{c}} X^{\dagger} X\right)$. The attached superscript "c" to the reduced density matrix is to specify that its evolution is conditioned on the measurement results. A simple ensemble average over a large number of particular realizations of $\rho^{\mathrm{c}}(t)$ would recover the unconditional density matrix $\rho(t)$ in Eq. (11), i.e. $\rho(t)=E\left[\rho^{\mathrm{c}}(t)\right]$, where $E[X]$ stands for an ensemble average of a large number of quantum trajectories. The involving stochastic variables for single electron tunneling events satisfy

$$
\begin{aligned}
E\left[\mathrm{~d} N_{\mathrm{L}}(t)\right] & =\mathcal{P}_{\mathrm{L}}(t) \mathrm{d} t=\operatorname{Tr}\left\{\mathcal{J}\left[\sqrt{\Gamma_{\mathrm{L}}}|1\rangle\langle 0|\right] \rho^{\mathrm{c}}\right\} \mathrm{d} t, \\
E\left[\mathrm{~d} N_{\mathrm{R}}(t)\right] & =\mathcal{P}_{\mathrm{R}}(t) \mathrm{d} t=\operatorname{Tr}\left\{\mathcal{J}\left[\sqrt{\Gamma_{\mathrm{R}}}|0\rangle\langle 1|\right] \rho^{\mathrm{c}}\right\} \mathrm{d} t .
\end{aligned}
$$

It is now clear that individual electron tunneling events condition the future evolution of the reduced density matrix [Eq. (20)], while instantaneous quantum state condi- tions the detected tunneling events through the left and right junctions [Eq. (21)]. By employing this approach, one thus is capable of propagating the conditioned quantum state $\left[\rho^{\mathrm{c}}(t)\right]$ and measurement result $\left[\mathrm{d} N_{\mathrm{L} / \mathrm{R}}(t)\right]$ in a self-consistent way.

The real-time quantum state $\left[\rho^{\mathrm{c}}(t)\right]$ and corresponding detection record of tunneling to the right electrode $\left[\mathrm{d} N_{\mathrm{R}}(t)\right]$ are plotted in Fig.3(a)-(d) for $\Omega / \Gamma=0.5$. For a give voltage $V / \Gamma=10$ in the bias regime 1 , the state " $|c\rangle$ " as shown in Fig.1(c) is energetically forbidden. When there is no extra electron in the TDQD, the qubit experiences some oscillations between the states "|a|" and "|b〉" shown in Fig. 1 with frequency $\sim \Omega$. Whenever one electron tunnels into the TDQD, the system collapses to the state "|d $\rangle$ ". The electron may stay in the double dot and experience some oscillations between QD1 and QD2, until it escapes to the right electrode. Correspond- 
ingly, the system jumps to the state "|b|", and an event of tunneling out to the right electrode is detected, i.e. $\mathrm{d} N_{\mathrm{R}}=1$. A typical example of the tunneling events is shown in Fig. 3 (d).

Very different tunneling behavior is observed in the case of a suppressed $\Omega$; see Fig. 33(e)-(h) for $\Omega / \Gamma=0.1$. One finds unambiguously the bunching of electron tunneling events though the TDQD. In most of the time, the

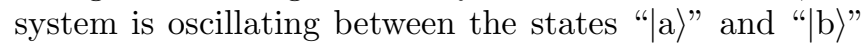
with a lower frequency $\sim \Omega$. Due to strong electrostatic interaction between the qubit and TDQD $(U / \Gamma \gg 1)$, the occupation of qubit in the logical state " $|\alpha\rangle$ " blocks the current through TDQD until it tunnels to the state " $|\beta\rangle$ ", which is then followed by a bunching of tunneling events through the TDQD during a short time window. It is right this mechanism that leads to the super-Poissonian Fano factor in Fig.2, Our result thus reveals that the intrinsic dynamics of the qubit may serves as an additional mechanism that may lead to dynamical blockade and eventually to the pronounced super-Poissonian behavior in noise spectrum.

Normally, detector shot noise leads to the dephasing of a qubit [19]. However, we will show in Sec.Vthat a large detector shot noise at small $\Omega$ does not necessarily imply a fast dephasing rate. In particular, it will be revealed that the dynamical blockade may have essential roles to play in the dephasing process of the qubit. The dephasing is suppressed at small $\Omega$, in spite of a large detector shot noise.

\section{DETECTION BACKACTION INDUCED DEPHASING}

To study the dephasing behavior of the qubit under continuous measurement of the TDQD detector, we shall make use of the density matrix of the qubit alone, which can be obtained by tracing out the degrees of freedom of the TDQD from the reduced (qubit plus TQDQ) density matrix

$$
\varrho(t)=\operatorname{tr}_{\mathrm{TDQD}}\{\rho(t)\},
$$

where $\operatorname{tr}_{\mathrm{TDQD}}\{\cdots\}$ stands for the trace over the degrees of freedom of the TDQD, and $\rho(t)$ is the unconditional density matrix that can be obtained from Eq. (11). To obtain time evolution of qubit state alone $\varrho(t)$, it is thus necessary to derive the equation of motion $\rho(t)$ first. In the state representation of Fig.1, the quantum master equation of $\rho(t)$ is given by

$$
\begin{gathered}
\dot{\rho}_{\mathrm{aa}}=\mathrm{i} \Omega\left(\rho_{\mathrm{ab}}-\rho_{\mathrm{ba}}\right)+\Gamma_{\mathrm{L}} \rho_{\mathrm{cc}}+\Gamma_{\mathrm{R}} \rho_{\mathrm{cc}}, \\
\dot{\rho}_{\mathrm{bb}}=\mathrm{i} \Omega\left(\rho_{\mathrm{ba}}-\rho_{\mathrm{ab}}\right)-\Gamma_{\mathrm{L}} \rho_{\mathrm{bb}}+\Gamma_{\mathrm{R}} \rho_{\mathrm{dd}}, \\
\dot{\rho}_{\mathrm{cc}}=\mathrm{i} \Omega\left(\rho_{\mathrm{cd}}-\rho_{\mathrm{dc}}\right)-\Gamma_{\mathrm{L}} \rho_{\mathrm{cc}}-\Gamma_{\mathrm{R}} \rho_{\mathrm{cc}}+\mathrm{i} W\left(\rho_{\mathrm{ce}}-\rho_{\mathrm{ec}}\right), \\
\dot{\rho}_{\mathrm{dd}}=\mathrm{i} \Omega\left(\rho_{\mathrm{dc}}-\rho_{\mathrm{cd}}\right)+\Gamma_{\mathrm{L}} \rho_{\mathrm{bb}}-\Gamma_{\mathrm{R}} \rho_{\mathrm{dd}}+\mathrm{i} W\left(\rho_{\mathrm{df}}-\rho_{\mathrm{fd}}\right), \\
\dot{\rho}_{\mathrm{ee}}=\mathrm{i} \Omega\left(\rho_{\mathrm{ef}}-\rho_{\mathrm{fe}}\right)-\mathrm{i} W\left(\rho_{\mathrm{ce}}-\rho_{\mathrm{ec}}\right), \\
\dot{\rho}_{\mathrm{ff}}=\mathrm{i} \Omega\left(\rho_{\mathrm{fe}}-\rho_{\mathrm{ef}}\right)-\mathrm{i} W\left(\rho_{\mathrm{df}}-\rho_{\mathrm{fd}}\right), \\
\dot{\rho}_{\mathrm{cd}}=\mathrm{i} \Omega\left(\rho_{\mathrm{cc}}-\rho_{\mathrm{dd}}\right)+\mathrm{i} W\left(\rho_{\mathrm{cf}}-\rho_{\mathrm{ed}}\right)-\mathrm{i} U \rho_{\mathrm{cd}}+\frac{1}{2} \Gamma_{\mathrm{L}}\left(\rho_{\mathrm{ab}}-\rho_{\mathrm{cd}}\right)-\Gamma_{\mathrm{R}} \rho_{\mathrm{cd}}, \\
\dot{\rho}_{\mathrm{ce}}=\mathrm{i} \Omega\left(\rho_{\mathrm{cf}}-\rho_{\mathrm{ed}}\right)+\mathrm{i} W\left(\rho_{\mathrm{cc}}-\rho_{\mathrm{ee}}\right)-\mathrm{i} U \rho_{\mathrm{ce}}-\frac{1}{2}\left(\Gamma_{\mathrm{L}}+\Gamma_{\mathrm{R}}\right) \rho_{\mathrm{ce}}, \\
\dot{\rho}_{\mathrm{cf}}=\mathrm{i} \Omega\left(\rho_{\mathrm{ce}}-\rho_{\mathrm{df}}\right)+\mathrm{i} W\left(\rho_{\mathrm{cd}}-\rho_{\mathrm{ef}}\right)-\mathrm{i} U \rho_{\mathrm{cf}}-\frac{1}{2}\left(\Gamma_{\mathrm{L}}+\Gamma_{\mathrm{R}}\right) \rho_{\mathrm{cf}}, \\
\dot{\rho}_{\mathrm{de}}=\mathrm{i} \Omega\left(\rho_{\mathrm{df}}-\rho_{\mathrm{ce}}\right)+\mathrm{i} W\left(\rho_{\mathrm{dc}}-\rho_{\mathrm{fe}}\right)-\frac{1}{2} \Gamma_{\mathrm{R}} \rho_{\mathrm{de}}, \\
\dot{\rho}_{\mathrm{df}}=\mathrm{i} \Omega\left(\rho_{\mathrm{de}}-\rho_{\mathrm{cf}}\right)+\mathrm{i} W\left(\rho_{\mathrm{dd}}-\rho_{\mathrm{ff}}\right)-\frac{1}{2} \Gamma_{\mathrm{R}} \rho_{\mathrm{df}}, \\
\dot{\rho}_{\mathrm{ef}}=\mathrm{i} \Omega\left(\rho_{\mathrm{ee}}-\rho_{\mathrm{ff}}\right)-\mathrm{i} W\left(\rho_{\mathrm{cf}}-\rho_{\mathrm{ed}}\right) .
\end{gathered}
$$

From the above coupled equations, one then is able to obtain the reduced dynamics of the qubit alone by using Eq. (22), i.e. $\varrho_{\alpha \alpha}=\rho_{\mathrm{aa}}+\rho_{\mathrm{cc}}+\rho_{\mathrm{ee}}, \varrho_{\beta \beta}=\rho_{\mathrm{bb}}+\rho_{\mathrm{dd}}+\rho_{\mathrm{ff}}$, and $\varrho_{\alpha \beta}=\rho_{\mathrm{ab}}+\rho_{\mathrm{cd}}+\rho_{\text {ef }}$, representing the probability of the qubit in the logical states $|\alpha\rangle,|\beta\rangle$, and linear superposition of the two logical states (so-called "quantum coherence"), respectively. By collecting relevant terms in Eq. (23), one eventually arrives at the equation of motion for the reduced density matrix of the qubit

$$
\begin{gathered}
\dot{\varrho}_{\alpha \alpha}(t)=\mathrm{i} \Omega\left(\varrho_{\alpha \beta}-\varrho_{\beta \alpha}\right), \\
\dot{\varrho}_{\beta \beta}(t)=\mathrm{i} \Omega\left(\varrho_{\beta \alpha}-\varrho_{\alpha \beta}\right), \\
\dot{\varrho}_{\alpha \beta}(t)=\mathrm{i} \Omega\left(\varrho_{\alpha \alpha}-\varrho_{\beta \beta}\right)-\mathrm{i} U \rho_{\mathrm{cd}} .
\end{gathered}
$$

Eqs. (24a) and (24b) denote coherent oscillations of the qubit, while Eq. (24c) stands for the dephasing of the qubit. Unambiguously, the qubit dephasing is directly related to $\rho_{\text {cd }}$ [cf. the last term in Eq. (24c)], which is 
further coupled to the dynamics of the entire system as shown in Eq. (23). It thus implies that the dynamics of the qubit and that of the TDQD are intimately entangled. Physically, due to detector current transport through two discrete levels of the TDQD, an electron tunneled into the TDQD is a linear superposition of these two states; the qubit itself is a two-state system described by superposition, leading thus eventually to the entanglement between the qubit and TDQD.

It was revealed that for a DQD detector, the qubit dephasing rate is directly related to the strength of the coupling between DQD and the left or right electrodes $\left(\Gamma_{\mathrm{L}}\right.$ or $\left.\Gamma_{\mathrm{R}}\right)$, rather than the interdot coupling of the qubit $(\Omega)$ [39]. We will show, however, the interdot coupling of the qubit may also have essential roles to play in the dephasing process of the qubit itself. It is thus instructive to study qubit dynamics at different values of $\Omega$. The numerical results, obtained by propagating Eqs. (23) and (24) in parallel, are displayed in Fig.4. Coherent oscillations of the qubit are shown in Fig.4(a) $\varrho_{\alpha \alpha}(t)$ and (b) $\varrho_{\beta \beta}(t)$, respectively. The dephasing of the qubit, described by the off-diagonal element of the reduced density matrix $\varrho_{\alpha \beta}(t)$, is plotted in Fig. 4 (c) for $\Omega / \Gamma=0.1$ (dashed curve) and $\Omega / \Gamma=1.0$ (solid curve). In both cases, $\varrho_{\alpha \beta}(t)$ vanishes in the long time limit, leading thus to the "collapse" of the reduced density matrix into the statistical mixture. However, the dephasing processes are indeed very different for the two cases. It is found via numerical fitting that the dephasing rate for $\Omega / \Gamma=1.0$ could reach almost 4 times larger that that for $\Omega / \Gamma=0.1$. Our result thus shows qubit interdot coupling $(\Omega)$ as an essential mechanism that may influence qubit dephasing, complementary to the conventional ways in SET measurement.

The unique suppression of the dephasing at small $\Omega$ can be interpreted as follows. In the case of a large interdot coupling $\Omega$ (for instance, $\Omega / \Gamma=1.0$ ), electrons tunnel through the TDQD very frequently; see individual electron tunneling events in Fig.2 (d). It thus gives rise to a frequent perturbation (measurement) of the qubit. In contrast, for a small $\Omega$ (cf. $\Omega / \Gamma=0.1$ ), electron transport through the TDQD is dynamically blockaded during the time windows where coherent oscillations between the states " $|\mathrm{a}\rangle$ " and "|b|" dominates, as shown in Fig.2(e)-(h). Yet, these time windows corresponds to no-measurement regimes where acquisition of qubit information is suppressed, leading eventually to the inhibition of the dephasing.

As is well known, the fundamental physics involved in quantum detection is the trade-off between acquisition of qubit information and the backaction-induced dephasing of the measured system. A question arises naturally for the present TDQD detector: Is the measurement more effective in the small $\Omega$ regime where dephasing is suppressed, or in the large $\Omega$ regime where the measurement takes place more frequently? Thus, we now investigate the effectiveness of the measurement at different values of qubit interdot coupling in Sec.VI

\section{MEASUREMENT EFFECTIVENESS}

A powerful tool to characterize the measurement effectiveness is the detector's noise spectrum $S(\omega)$. The qubit oscillations are manifested in $S(\omega)$ as a peak located at the qubit characteristic frequency $\omega_{\mathrm{c}}=\left(\epsilon_{\mathrm{q}}^{2}+4 \Omega^{2}\right)^{1 / 2}$. An essential feature of this peak is that its height with respective to the pedestal, also known as signal-to-noise ratio, provides a measure of detector's effectiveness, showing how close to the quantum limit the detector may operate [26, 27]. It was argued that for any linear-response detectors there is a fundamental limit imposed on the signal-to-noise ratio, i.e. the so-called Korotkov-Averin bound [26, 27]: The peak height can reach maximally 4 times the noise pedestal for an ideal or quantum-limited detector. In contrast, for a less efficient detector the qubit dephasing takes place more rapidly than information acquisition, and the signal-to-noise ratio is less than four.

To analyze the signal-to-noise ratio of a TDQD detector, we first study the noise spectrum of the tunneling currents through the left or right junction $S_{\mathrm{L} / \mathrm{R}}(\omega)$ [cf. Eq. (15)]. The numerical result is displayed in Fig.5(a), where the noise of the tunneling current is scaled by its own pedestal

$$
S_{\mathrm{L} / \mathrm{R}}(\infty)=\frac{2 \Gamma_{\mathrm{L}} \Gamma_{\mathrm{R}}}{3 \Gamma_{\mathrm{L}}+2 \Gamma_{\mathrm{R}}} .
$$

The noises of left and right junction currents are found to be consistent within the whole frequency regime, i.e. $S_{\mathrm{L}}(\omega)=S_{\mathrm{R}}(\omega)$. The noise at various values of interdot couplings $(\Omega)$ is plotted in Fig.5(a). The peaks in vicinity of $\omega \approx 2 \Omega$ reflect signal of qubit coherent oscillations. The peak width increases with rising $\Omega$, indicating the enhancement of the dephasing rate. It thus confirms our previous argument of the dependence of the dephasing on qubit interdot coupling.

What we are most interested is the height of the peak of qubit oscillations, which provides the measure of signalto-noise ratio of quantum measurement. For the present TDQD detector, it is found in Fig.5 that the peak height at different values of $\Omega$ does not show striking difference. Although $\Omega$ has essential roles to play in the dephasing of the qubit, its influence on signal-to-noise ratio is very limited. At small $\Omega$, dephasing is inhibited but information acquisition is also suppressed. An increase of $\Omega$ leads to fast information gain, whereas the qubit lose coherence more rapidly. Eventually, the measurement effectiveness turns out to be insensitive to the qubit interdot coupling $(\Omega)$. Furthermore, by considering the noise spectrum of the tunneling currents, the signal-to-noise is found to be well below the Korotkov-Averin bound. This is qualitatively consistent with the result in Ref. 40, where the signal-to-noise ratio of a serial DQD detector is found below " 4 ". It might lead us to conclude that neither the TDQD nor the serial DQD can reach the effectiveness of an ideal detector, if one takes solely the tunneling current noise into consideration.

However, this picture is not yet complete for a TDQD 

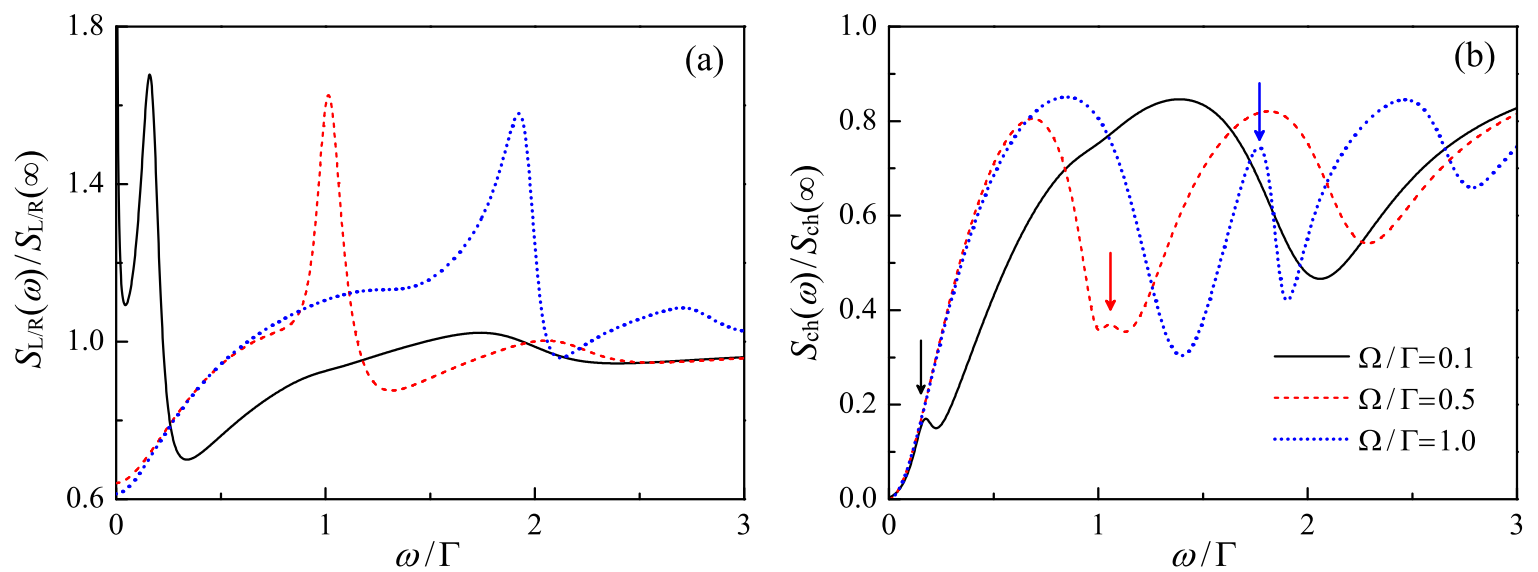

FIG. 5: (a) Noise spectrum of the tunneling current through the left or right electrodes $S_{\mathrm{L} / \mathrm{R}}(\omega)$, scaled by its own pedestal $S_{\mathrm{L} / \mathrm{R}}(\infty)$. (b) Spectrum of charge fluctuation $S_{\mathrm{ch}}(\omega)$ in the TDQD with respective to its pedestal $S_{\mathrm{ch}}(\infty)$. The total tunneling width $\Gamma=\Gamma_{\mathrm{L}}+\Gamma_{\mathrm{R}}$ is kept constant. Other parameters are: $\epsilon_{\mathrm{q}}=\epsilon_{\mathrm{T}}=0, W / \Gamma=1.0, V / \Gamma=10$, and $U / \Gamma=10$.

detector, since the currents through the left and right junctions are intrinsically correlated via the charge accumulation in the TDQD, owing to the condition of charge conservation [cf. Eq. (12)]. The noise of the circuit current is actually a superposition of each component; see Eq. (14). It is thus of importance to study the influence of charge fluctuation $\left[S_{\mathrm{ch}}(\omega)\right]$ on the signal-to-noise ratio. In particular, we will show in Sec.VII that under appropriate conditions the charge fluctuation leads to a strong suppression of the noise pedestal. It gives rise to a strong enhancement of the signal-to-noise ratio, leading eventually to the violation of the Korotkov-Averin bound.

\section{VIOLATION OF THE KOROTKOV-AVERIN BOUND}

Fig. 5 (b) shows the numerical result of the charge fluctuations in the TDQD for various values of $\Omega$. The plot of the charge fluctuation is scaled by its own pedestal

$$
S_{\mathrm{ch}}(\infty)=\frac{8 \Gamma_{\mathrm{L}} \Gamma_{\mathrm{R}}}{3 \Gamma_{\mathrm{L}}+2 \Gamma_{\mathrm{R}}} .
$$

In the low frequency limit, the charge fluctuation in the TDQD is strongly inhibited, as implied in Eq. (17). The basic signals are the peaks located in the vicinity of frequency $2 \Omega$, indicating qubit coherent oscillations; see the arrows in Fig.5(b).

The charge fluctuation may have a significant impact on the signal-to-noise ratio of the measurement, as displayed in Fig.6. In case of very asymmetric junction capacitances, for instance, $\eta_{\mathrm{L}}: \eta_{\mathrm{R}}=9: 1$ or $\eta_{\mathrm{L}}: \eta_{\mathrm{R}}=8: 2$, the spectrum of charge fluctuations has only very limited contribution to the circuit noise [cf. Eq. (14)]. The resultant signal-to-noise ratio is below the Korotkov-Averin bound; see the solid and dashed curves in Fig.6. However, when the junction capacitances get more and more symmetric, the charge fluctuation may have a vital role to play in the noise of circuit current. Strikingly, for $\eta_{\mathrm{L}}: \eta_{\mathrm{R}}=7: 3$, a prominent enhancement of the signalto-noise ratio is observed and the signal-to-noise ratio exceeds the upper limit of " 4 ", i.e. the violation of the Korotkov-Averin bound. Furthermore, by checking the pedestals of the tunneling current noise [Eq. (25)] and charge fluctuation [Eq. (26)], one finds that for symmetric junction capacitances $\left(\eta_{\mathrm{L}}: \eta_{\mathrm{R}}=1: 1\right)$, the pedestal of the circuit noise [Eq. (14)] can be completely eliminated, resulting thus in a divergence of the signal-to-noise ratio. Our new finding thus provides a transparent and direct way to improve the signal-to-noise ratio of a TDQD detector.

In literature, different approaches have been proposed that may lead to the violation of the Korotkov-Averin bound. Normally, they fall into two categories. The first type is based on the enhancement of measurement signal by employing approaches such as quantum nondemolition measurements [70, 71], non-Markovian memory effect [72], or quantum feedback scheme [73, 74]. The second on concerns with the reduction of the noise pedestal by utilizing twin detectors 45, , 75], or strongly responding detectors [20]. The occurrence of a divergent signalto-noise ratio in this work arises from a complete suppression of the noise pedestal. Our result shows that by simply adjusting the junction capacitances, one may considerably enhance the signal-to-noise ratio of a TDQD detector in quantum measurement.

\section{SUMMARY}

We have proposed to continuously monitor a charge qubit by utilizing a T-shaped double quantum dot detector, in which only one dot is directly tunnel-coupled to the electrodes. It is demonstrated that the dynam- 


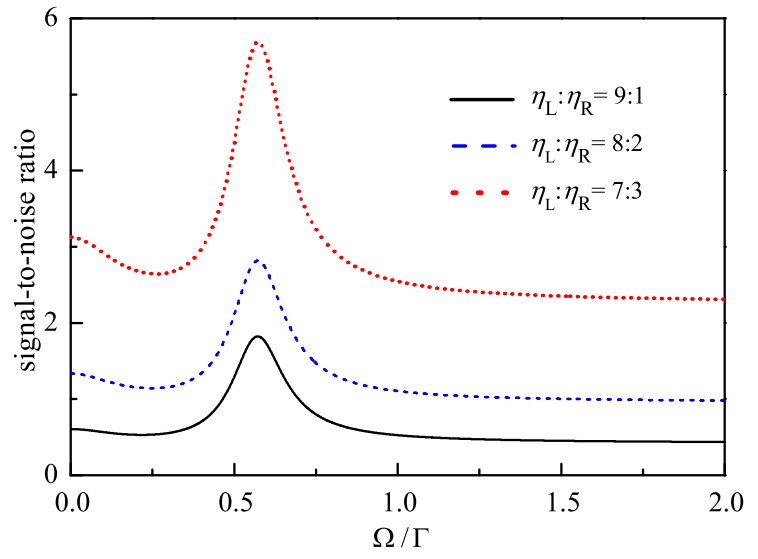

FIG. 6: The signal-to-noise ratio versus $\Omega$ obtained from the circuit current noise with different configurations of left and right junction capacitances $\left(\eta_{\mathrm{L}}: \eta_{\mathrm{R}}\right)$. The coefficients satisfies $\eta_{\mathrm{L}}+\eta_{\mathrm{R}}=1$. The total tunneling width $\Gamma=\Gamma_{\mathrm{L}}+\Gamma_{\mathrm{R}}$ is kept constant. Other plotting parameters used are: $\epsilon_{\mathrm{q}}=\epsilon_{\mathrm{T}}=0$, $W / \Gamma=1.0, V / \Gamma=10, \Gamma_{\mathrm{L}} / \Gamma_{\mathrm{R}}=0.5$, and $U / \Gamma=10$.

ics of the qubit and the detector output are intrinsically correlated. In case of a suppressed interdot coupling between the two states of the qubit, a dynamical block- ade mechanism takes place, leading to a super-Poisson shot noise. However, such a pronounced enhancement of the noise does not necessarily produce a fast dephasing rate. Actually, an inhibited dephasing is observed, since the involving dynamical blockade is directly related to the regime where no information is acquired. The major advantage of the present T-shaped double quantum dot detector is that its spectrum of charge fluctuations may significantly suppress the pedestal of the circuit noise. Remarkably, the noise pedestal could be removed completely under the condition of symmetric junction capacitances, leading to a divergent signal-to-noise ratio, and eventually to the violation the Korotkov-Averin bound in quantum measurement. The proposed TDQD thus may serve as an essential candidate detector to reach the quantum limited effectiveness in a very transparent and straightforward manner.

\section{Acknowledgments}

Support from the National Natural Science Foundation of China (11147114 and 11204272) and the Natural Science Foundation of Zhejiang Province (Y6110467) are gratefully acknowledged.
[1] A. E. Allahverdyan, R. Balian, and T. M. Nieuwenhuizen, Phys. Rep. 525, 1 (2013).

[2] H. M. Wiseman and G. J. Milburn, Quantum Measurement and Control (Cambridge University Press, Cambridge, 2010).

[3] J. R. Petta, A. C. Johnson, J. M. Taylor, E. A. Laird, A. Yacoby, M. D. Lukin, C. M. Marcus, M. P. Hanson, and A. C. Gossard, Science 309, 2180 (2005).

[4] A. A. Clerk, S. M. Girvin, and A. D. Stone, Phys. Rev. B 67, 165324 (2003).

[5] S. Pilgram and M. Büttiker, Phys. Rev. Lett. 89, 200401 (2002).

[6] D. V. Averin and E. V. Sukhorukov, Phys. Rev. Lett. 95, 126803 (2005).

[7] X. Q. Li, P. Cui, and Y. J. Yan, Phys. Rev. Lett. 94, 066803 (2005).

[8] D. Taubert, M. Pioro-Ladrière, D. Schröer, D. Harbusch, A. S. Sachrajda, and S. Ludwig, Phys. Rev. Lett. 100, 176805 (2008).

[9] J. Y. Luo, H. J. Jiao, F. Li, X.-Q. Li, and Y. J. Yan, J. Phys.: Cond. Matt. 21, 385801 (2009).

[10] S. Gustavsson, R. Leturcq, M. Studer, I. Shorubalko, T. Ihn, K. Ensslin, D. Driscoll, and A. Gossard, Surf. Sci. Rep. 64, 191 (2009).

[11] K. D. Petersson, J. R. Petta, H. Lu, and A. C. Gossard, Phys. Rev. Lett. 105, 246804 (2010).

[12] C. E. Young and A. A. Clerk, Phys. Rev. Lett. 104, 186803 (2010).

[13] M. Thomas and A. Romito, Phys. Rev. B 86, 235419 (2012).

[14] N. Ubbelohde, C. Fricke, C. Flindt, F. Hohls, and R. J.
Haug, Nature Communications 3, 612 (2012).

[15] M. H. Devroret and R. J. Schoelkopf, Nature 406, 1039 (2000).

[16] Y. Makhlin, G. Schön, and A. Shnirman, Rev. Mod. Phys. 73, 357 (2001).

[17] A. A. Clerk, S. M. Girvin, A. K. Nguyen, and A. D. Stone, Phys. Rev. Lett. 89, 176804 (2002).

[18] D. Mozyrsky, I. Martin, and M. B. Hastings, Phys. Rev. Lett. 92, 018303 (2004).

[19] S. A. Gurvitz and G. P. Berman, Phys. Rev. B 72, 073303 (2005).

[20] H. Jiao, F. Li, S.-K. Wang, and X.-Q. Li, Phys. Rev. B 79, 075320 (2009).

[21] J. Y. Luo, H. J. Jiao, J. Z. Wang, Y. Shen, and X.-L. He, Phys. Lett. A 374, 4904 (2010).

[22] W. Lu, Z. Ji, L. Pfeiffer, K. W. West, and A. J. Rimberg, Nature 423, 422 (2003).

[23] M. J. Biercuk, D. J. Reilly, T. M. Buehler, V. C. Chan, J. M. Chow, R. G. Clark, and C. M. Marcus, Phys. Rev. B 73, 201402 (2006).

[24] A. Morello, J. J. Pla, F. A. Zwanenburg, K. W. Chan, K. Y. Tan, H. Huebl, M. Möttönen, C. D. Nugroho, C. Yang, J. A. van Donkelaar, et al., Nature 467, 687 (2010).

[25] J. P. Dehollain, J. T. Muhonen, K. Y. Tan, A. Saraiva, D. N. Jamieson, A. S. Dzurak, and A. Morello, Phys. Rev. Lett. 112, 236801 (2014).

[26] A. N. Korotkov and D. V. Averin, Phys. Rev. B 64, 165310 (2001).

[27] A. N. Korotkov, Phys. Rev. B 63, 085312 (2001).

[28] R. Ruskov and A. N. Korotkov, Phys. Rev. B 67, 075303 
(2003).

[29] A. Shnirman, D. Mozyrsky, and I. Martin, Europhys. Lett. 67, 840 (2004).

[30] E. Il'ichev, N. Oukhanski, A. Izmalkov, T. Wagner, M. Grajcar, H.-G. Meyer, A. Y. Smirnov, A. M. van den Brink, M. H. S. Amin, and A. M. Zagoskin, Phys. Rev. Lett. 91, 097906 (2003).

[31] W. J. Mao, D. V. Averin, R. Ruskov, and A. N. Korotkov, Phys. Rev. Lett. 93, 056803 (2004).

[32] W. J. Mao, D. V. Averin, F. Plastina, and R. Fazio, Phys. Rev. B 71, 085320 (2005).

[33] L. N. Bulaevskii and G. Ortiz, Phys. Rev. Lett. 90, 040401 (2003).

[34] Z. Nussinov, M. F. Crommie, and A. V. Balatsky, Phys. Rev. B 68, 085402 (2003).

[35] R. Schleser, E. Ruh, T. Ihn, K. Ensslin, D. C. Driscoll, and A. C. Gossard, Appl. Phys. Lett. 85, 2005 (2004).

[36] L. M. K. Vandersypen, J. M. Elzerman, R. N. Schouten, L. H. W. van Beveren, R. Hanson, and L. P. Kouwenhoven, Appl. Phys. Lett. 85, 4394 (2004).

[37] S. Gustavsson, R. Leturcq, B. Simovic, R. Schleser, T. Ihn, P. Studerus, K. Ensslin, D. C. Driscoll, and A. C. Gossard, Phys. Rev. Lett. 96, 076605 (2006).

[38] T. Fujisawa, T. Hayashi, R. Tomita, and Y. Hirayama, Science 312, 1634 (2006).

[39] H. Jiao, X.-Q. Li, and J. Y. Luo, Phys. Rev. B 75, 155333 (2007).

[40] T. Gilad and S. A. Gurvitz, Phys. Rev. Lett. 97, 116806 (2006).

[41] J. Y. Luo, S.-K. Wang, X.-L. He, X.-Q. Li, and Y. J. Yan, J. Appl. Phys. 108, 083720 (2010).

[42] J. Y. Luo, Y. Shen, X.-L. He, X.-Q. Li, and Y. J. Yan, Phys. Lett. A 376, 59 (2011).

[43] J. Y. Luo, H. J. Jiao, B. T. Xiong, X.-L. He, and C. Wang, J. Phys.: Condens. Matter 25, 155304 (2013).

[44] H.-B. Xue, Ann. Phys. 339, 208 (2013).

[45] A. N. Jordan and M. Büttiker, Phys. Rev. Lett. 95, 220401 (2005).

[46] Y. M. Blanter and M. Büttiker, Phys. Rep. 336, 1 (2000).

[47] Y. V. Nazarov(Ed.), Quantum Noise in Mesoscopic Physics (Kluwer Academic Publishers, Dordrecht, 2003).

[48] S. A. Gurvitz and Y. S. Prager, Phys. Rev. B 53, 15932 (1996).

[49] R. Aguado and T. Brandes, Phys. Rev. Lett. 92, 206601 (2004).

[50] J. Y. Luo, X.-Q. Li, and Y. J. Yan, J. Phys.: Cond. Matt. 20, 345215 (2008).

[51] F. Li, H. J. Jiao, J. Y. Luo, X.-Q. Li, and S. A. Gurvitz, Physica E 41, 1707 (2009).
[52] C. Emary and R. Aguado, Phys. Rev. B 84, 085425 (2011).

[53] C. Flindt, T. Novotný, A. Braggio, M. Sassetti, and A.-P. Jauho, Phys. Rev. Lett. 100, 150601 (2008).

[54] A. Braggio, J. König, and R. Fazio, Phys. Rev. Lett. 96, 026805 (2006).

[55] J. Y. Luo, X.-Q. Li, and Y. J. Yan, Phys. Rev. B 76, 085325 (2007).

[56] S. A. Gurvitz, D. Mozyrsky, and G. P. Berman, Phys. Rev. B 72, 205341 (2005).

[57] D. K. C. MacDonald, Noise and Fluctuations: An Introduction (Wiley, New York, 1962), ch. 2.2.1.

[58] C. Flindt, T. Novotný, and A.-P. Jauho, Physica E 29, 411 (2005).

[59] D. Urban and J. König, Phys. Rev. B 79, 165319 (2009).

[60] H.-F. Lu, J.-R. Zhang, T. Wu, X.-T. Zu, and H.-W. Zhang, J. Appl. Phys. 107, 034314 (2010).

[61] J. Y. Luo, H. J. Jiao, Y. Shen, G. Cen, X.-L. He, and C. Wang, J. Phys.: Condens. Matter 23, 145301 (2011).

[62] R.-Q. Wang, L. Sheng, L.-B. Hu, B. G. Wang, and D. Y. Xing, Phys. Rev. B 84, 115304 (2011).

[63] B. H. Wu and J. C. Cao, Phys. Rev. B 81, 125326 (2010).

[64] J. Y. Luo, J. Hu, X. L. Lang, Y. Shen, X.-L. He, and H. J. Jiao, Phys. Lett. A 378, 892 (2014).

[65] N. Ubbelohde, C. Fricke, F. Hohls, and R. J. Haug, Phys. Rev. B 88, 041304 (2013).

[66] M. Misiorny, I. Weymann, and J. Barnaś, Phys. Rev. B 79, 224420 (2009).

[67] I. Weymann, B. R. Bułka, and J. Barnaś, Phys. Rev. B 83, 195302 (2011).

[68] Y. Okazaki, S. Sasaki, and K. Muraki, Phys. Rev. B 87, 041302 (2013).

[69] H. S. Goan, G. J. Milburn, H. M. Wiseman, and H. B. Sun, Phys. Rev. B 63, 125326 (2001).

[70] D. V. Averin, Phys. Rev. Lett. 88, 207901 (2002).

[71] A. N. Jordan and M. Büttiker, Phys. Rev. B 71, 125333 (2005).

[72] J. Y. Luo, H. J. Jiao, B. T. Xiong, X.-L. He, and C. R. Wang, J. Appl. Phys. 114, 173703 (2013).

[73] S.-K. Wang, J. S. Jin, and X.-Q. Li, Phys. Rev. B 75, 155304 (2007).

[74] R. Vijay, C. Macklin, D. H. Slichter, S. J. Weber, K. W. Murch, R. Naik, A. N. Korotkov, and I. Siddiqi, Nature 490, 77 (2012).

[75] B. Küng, O. Pfäffli, S. Gustavsson, T. Ihn, K. Ensslin, M. Reinwald, and W. Wegscheider, Phys. Rev. B 79, 035314 (2009). 